\title{
Arte e Saúde: Frequência de Sintomas Músculo-Esqueléticos em Músicos da Orquestra Sinfônica da Universidade Estadual de Londrina ${ }^{1}$
}

\section{Art and Health: Frequency of Musculoskeletal Symptoms in Musicians of the Symphonic Orchestra of the State University of Londrina}

\author{
Celita Salmaso Trelha ${ }^{2}$; Renata Pagung de Carvalho ${ }^{3}$; Simone Silveira Franco ${ }^{3}$; \\ Tatiana Nakaoski ${ }^{3}$; Thayza Priscilla Broza ${ }^{3}$; Thiago de Lorena Fábio ${ }^{3}$; \\ Thiago Zoratti Abelha ${ }^{3}$
}

\begin{abstract}
Resumo
Este estudo teve o propósito de verificar a freqüência de sintomas músculo-esqueléticos em músicos da Orquestra Sinfônica da Universidade Estadual de Londrina. Participaram do estudo 45 músicos que responderam um questionário auto-aplicável, que trata dos seguintes aspectos: dados pessoais, atividades profissionais, sintomatologia relacionada ao trabalho, tratamento realizado e consequiências da sintomatologia. Para a análise das variáveis, foram utilizados o Teste de qui-quadrado com correção de Yates e o Teste de Fisher. Dos 45 músicos estudados, 82,2\% constituíram-se indivíduos do gênero masculino e com uma média de idade de 39,56 anos. Do total de músicos pesquisados, 77,8\% relataram apresentar sintomas músculo-esqueléticos nos últimos doze meses e 71,1\% nos últimos sete dias. As regiões anatômicas mais acometidas foram: ombros, coluna cervical, coluna dorsal e punhos e mãos. Verificou-se um maior predomínio de sintomatologia em músicos que tocam corda e sopro. Em decorrência da sintomatologia apresentada, $33,3 \%$ dos profissionais relataram ter perdido dias de trabalho. O presente estudo encontrou uma elevada prevalência de sintomas músculo-esqueléticos em músicos da OSUEL, com referência tanto aos últimos doze meses quanto aos últimos sete dias precedentes à auto-aplicação do questionário, principalmente na região de coluna e ombros. Esses sintomas podem estar relacionados às cargas físicas e emocionais da própria atividade profissional. Diante dos resultados encontrados, faz-se necessária a elaboração e implantação de estratégias para amenizar a carga de trabalho e evitar agravos.

Palavras-chave: Saúde do trabalhador. Músico. Sintoma Músculo-esquelético.
\end{abstract}

1 Trabalho recebeu Menção Honrosa no I Congresso de Fisioterapia Ortopédica e Traumatológica da UEL.

2 Fisioterapeuta, Docente do Departamento de Fisioterapia da Universidade Estadual de Londrina, Mestre em Saúde Coletiva. Centro -Londrina PR - e-mail: celita@uel.br

3 Acadêmicos do Curso de Fisioterapia da Universidade Estadual de Londrina. 


\begin{abstract}
This study had the purpose of determining the frequency of musculoskeletal symptoms in musicians of the Symphonic Orchestra of the State University of Londrina. 45 musicians took part in this study answering a self-administered questionnaire with the following aspects: personal data, job-factor survey, symptom related to work, treatment and its consequences. As for the analysis of the variables the quisquare Test was used with Yates correction and the Test of Fisher. Of the 45 musicians studied, 82,2\% were men and with an average age of 39,56 years old. Of all the researched musicians, $77,8 \%$ reported the presence of musculoskeletal symptoms during the last twelve months and $71,1 \%$ in the last seven days. The highest prevalence was in the following anatomical areas: shoulders, neck, spine, hands and wrists. The present study found a high prevalence of musculoskeletal symptoms in musicians who play string and wind instruments. Due to the symptoms, 33,3\% of the professionals lost days of work. The present study found a high prevalence of musculoskeletal symptoms not only during the last twelve months but also during the last seven days that preceded the answering of the questionnaire, mainly in the spine and shoulders area. These symptoms can be related to physical and emotional loads. Taking the results into account, the elaboration and implantation of strategies to soften the workload and to avoid disorders are necessary.
\end{abstract}

Key words: Worker's health. Musician. Musculoskeletal Symptoms.

\section{Introdução}

A música sempre está associada ao bem estar, equilíbrio emocional e divertimento. Ao pensar em música, é difícil lembrar de doenças, dores e fatores de riscos. Atualmente, são crescentes as evidências de que os músicos encontram-se expostos a fatores de risco do próprio trabalho, propiciando o aparecimento de lesões.

Segundo Lockwood (1989) e Hoppmann (2001), as lesões que mais acometem os músicos profissionais são as desordens músculo-esqueléticas, principalmente as Lesões por Esforços Repetitivos/ Distúrbios Osteomusculares Relacionados ao Trabalho (LER/DORT). Essas lesões são definidas como um conjunto de afecções que podem acometer o sistema músculo-esquelético, isolada ou associadamente, com ou sem degeneração de tecidos, atingindo principalmente, membros superiores, região escapular e pescoço e de origem ocupacional (BRASIL, 2000).

Os músicos podem adquirir a doença por realizar movimentos repetitivos em posturas inadequadas e vivenciar situações de estresse (FRY, 1986; LOCKWOOD, 1989; ZAZA; CHARLES; MUSZYNSKI, 1998; PARASURAMAN; PUROHIT, 2000). Músicos de todas as idades e habilidades podem ser acometidos pelo agravo (LIU; HAYDEN, 2002).

Estudos epidemiológicos confirmam a severidade do problema. Brandfonbrener (1990) refere que os músicos de orquestras são os mais afetados por desordens músculo-esqueléticas. Em um estudo retrospectivo com o propósito de conhecer as razões porque os músicos procuravam médicos ortopedistas no período de 1994 a 2001, os autores verificaram que dois terços do total de 227 músicos apresentavam alguma desordem do sistema músculo-esquelético (NOURISSAT; CHAMAGNE; DUMONTIER, 2003). Fishbein et al. (1988) analisaram 4000 músicos de orquestras nos Estados Unidos e verificaram uma elevada prevalência de sintomas músculoesqueléticos. Na Espanha, Rosety-Rodiguez (2003) verificou que $65 \%$ dos pianistas analisados apresentavam ou apresentaram sintomas músculoesqueléticos e muitos destes eram bem jovens. Segundo Zaza (1998), a prevalência desses sintomas em músicos é semelhante a de outras categorias profissionais como operadores de checkouts e empacotadores.

Os sintomas músculo-esqueléticos variam desde um desconforto que pode persistir, piorar e levar a lesões sérias e conseqüente inabilidade e afastamento 
da profissão (AMADIO; RUSSOTTI, 1990). Também são registradas outras repercussões, como a interrupção da rotina doméstica (RANNEY, 2000).

Segundo Brito, Orso e Gomes (1992), essas lesões podem se apresentar sob várias formas, como afecções musculotendíneas, compressões nervosas ou disfunções motoras e são passíveis de tratamento efetivo quando iniciado precocemente.

Este estudo teve o propósito de verificar a freqüência de sintomas músculo-esqueléticos em músicos da Orquestra Sinfônica da Universidade Estadual de Londrina (OSUEL).

\section{Materiais e Métodos}

Para atingir o objetivo proposto, foi realizado um estudo transversal, com 45 músicos da OSUEL. A orquestra é composta de 49 músicos. Deste total 4 músicos não responderam o questionário, obtendo portanto um retorno de $91,8 \%$.

O Instrumento de coleta de dados baseou-se no Nordic Questionnaire (KUORINKA; JONSSON; KILBOM, 1987), traduzido e validado para sintomas osteomusculares relacionados ao trabalho para a população brasileira (PINHEIRO; TRÓCCOLI; CARVALHO, 2002). O questionário tratou dos seguintes aspectos:

1) dados pessoais: gênero, idade e estado civil;

2) atividades profissionais: tempo na orquestra, tipo de instrumento e carga horária de trabalho;

3) sintomatologia: ocorrência de dores ou desconforto, relacionados ao trabalho, em 10 regiões corporais, em relação aos últimos doze meses e últimos sete dias precedentes à auto-aplicação do questionário, diagnóstico médico, tratamento realizado, dias de trabalho perdidos e incapacidade de realizar alguma atividade, mudança nas atividades.
Para o tratamento estatístico dos dados, foi utilizado o programa Epi Info 6.04b e software Excel da Microsoft. Para a análise das variáveis foram utilizados o Teste de qui-quadrado com correção de Yates e o Teste de Fisher nos casos em que ocorreram freqüência esperada menor que 5 . O nível de significância adotado foi de 5\% (SOARES; SIQUEIRA, 1999).

\section{Resultados e Discussão}

A população estudada constituiu-se predominantemente por profissionais do gênero masculino. Dos 45 músicos estudados, $37(82,2 \%)$ foram indivíduos do gênero masculino e $8(17,8 \%)$ do gênero feminino. A idade variou de 21 a 66 anos, com média de 39,56 \pm 9,81 e uma distribuição mais detalhada pode ser observada na Figura 1. Em relação ao estado civil, verificou-se que $33(73,33 \%)$ referiram ser casados, $10(22,4 \%)$ solteiros e 2 $(4,44 \%)$ divorciados.

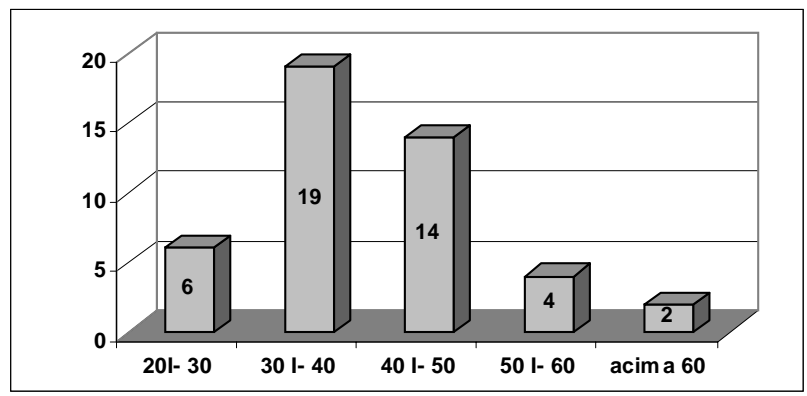

Figura 1. Distribuição dos Músicos da OSUEL segundo a faixa etária em anos.

O tipo de instrumento que os profissionais referiram tocar foram: sopro, metal, corda, percussão e teclado e essa distribuição pode ser observada na Tabela 1. 
Tabela 1. Distribuição dos músicos da Orquestra Sinfônica da UEL segundo o instrumento musical.

\begin{tabular}{ll|ll}
\hline Instrumento Musical & & n \\
& & & \\
\hline Sopro & Clarinete & $\mathbf{2}$ & $\mathbf{4 , 4}$ \\
& Fagote & $\mathbf{1}$ & $\mathbf{2 , 2}$ \\
& Flauta transversal & $\mathbf{3}$ & $\mathbf{6 , 6}$ \\
& Oboé & $\mathbf{3}$ & $\mathbf{6 , 6}$ \\
& Trombone & $\mathbf{3}$ & $\mathbf{6 , 6}$ \\
Metal & Trompa & $\mathbf{3}$ & $\mathbf{6 , 6}$ \\
& Trompete & $\mathbf{3}$ & $\mathbf{6 , 6}$ \\
& Tuba & $\mathbf{1}$ & $\mathbf{2 , 2}$ \\
& Contrabaixo & $\mathbf{4}$ & $\mathbf{8 , 9}$ \\
Corda & Viola & $\mathbf{5}$ & $\mathbf{1 1 , 1}$ \\
& Violino & $\mathbf{1 1}$ & $\mathbf{2 4 , 4}$ \\
& Violoncelo & $\mathbf{2}$ & $\mathbf{4 , 4}$ \\
& Percussão & $\mathbf{3}$ & $\mathbf{6 , 6}$ \\
Percussão & & $\mathbf{1}$ & $\mathbf{2 , 2}$ \\
& Piano & $\mathbf{4 5}$ & $\mathbf{1 0 0 , 0}$ \\
Teclado & & \multicolumn{2}{c}{} \\
Total & &
\end{tabular}

Os músicos da OSUEL referiram trabalhar na orquestra em média 31,85 horas semanais $\pm 9,26$ e o tempo de atuação na orquestra variou de 0,75 a 20 anos, com média de 12,67 $\pm 4,84$.

Dos 45 músicos pesquisados, 35 (77,8\%) relataram apresentar algum sintoma músculo-esquelético relacionado ao trabalho nos últimos doze meses e ainda dos 45 músicos, $32(71,1 \%)$ relataram apresentar sintomas nos últimos sete dias precedentes à autoaplicação do questionário. As regiões anatômicas mais acometidas, com referência nos últimos doze meses, foram: ombro $(48,9 \%)$, coluna cervical $(46,7 \%)$ e coluna dorsal $(46,7 \%)$. Analisando os últimos sete dias observou-se que as regiões acometidas foram: ombro $(44,4 \%)$, coluna cervical $(40,0 \%)$ e coluna dorsal $(40,0 \%)$ como pode ser observado na Tabela 2 .

Tabela 2. Prevalência de sintomas músculo-esqueléticos em Músicos da OSUEL por localização anatômica nos últimos doze meses e últimos sete dias.

\begin{tabular}{l|l|l|l|l}
\hline \multirow{2}{*}{ REGIÃO ANATÔMICA } & \multicolumn{4}{l}{ SINTOMAS MÚSCULO-ESQUELÉTICOS } \\
\cline { 2 - 6 } & \multicolumn{1}{l}{$\begin{array}{l}\text { ÚLTIMOS } \\
\text { DOZE MESES }\end{array}$} & \multicolumn{2}{l}{ ÚLTIMOS SETE DIAS } \\
\cline { 2 - 5 } & $\mathrm{n}$ & $\%$ & $\mathrm{n}$ & $\%$ \\
\hline Ombros & 22 & 48,9 & 20 & 44,4 \\
\hline Coluna Cervical & 21 & 46,7 & 18 & 40,0 \\
\hline Coluna Dorsal & 21 & 46,7 & 18 & 40,0 \\
\hline Punhos e Mãos & 15 & 33,3 & 14 & 31,1 \\
\hline Coluna Lombar & 7 & 15,6 & 7 & 15,6 \\
\hline Cotovelos & 5 & 11,1 & 5 & 11,1 \\
\hline Dedos & 4 & 8,9 & 4 & 8,9 \\
\hline Joelhos & 2 & 4,4 & 1 & 2,2 \\
\hline Pés e Tornozelos & 1 & 2,2 & 1 & 2,2 \\
\hline Quadril & - & - & - & - \\
\hline
\end{tabular}

Os Músicos relataram sintomas músculoesqueléticos em mais de uma região anatômica, por este motivo o número e o percentual apresentado correspondem às respostas obtidas e não ao número da amostra.

O presente estudo encontrou uma elevada freqüência de sintomas músculo-esqueléticos em músicos da OSUEL. Os dados coadunam com a literatura. Joubrel et al. (2001) verificaram entre 141 músicos instrumentais franceses que $76,6 \%$ apresentavam sintomas músculo-esqueléticos, principalmente em coluna, punho e mão. Siemon e Borisch (2002) observaram que $84 \%$ dos 130 músicos de orquestra estudados apresentaram problemas de saúde e $74 \%$ estava relacionado ao sistema músculoesquelético, principalmente em ombros, pescoço, coluna lombar, e mãos. Índices elevados também foram observados por Krapac (2001) em 40 músicos que tocavam metal.

Lockwood (1989) refere que mãos, ombros e cabeça são as regiões anatômicas mais comumente acometidas. Toledo et al. (2004) também referem que os membros superiores são os segmentos mais afetados.

Segundo Bejjani, Kaye e Benham (1996), os sintomas músculo-esqueléticos nos músicos estão associados aos movimentos repetitivos ao tocar associados a postura inadequada durante tempo prolongado para manter o instrumento. $\mathrm{O}$ autor refere-se também à forma e peso dos instrumentos.

Em relação a sintomatologia apresentada e o tipo de instrumento, não foi encontrada diferença significativa, porém verificou-se um maior predomínio de sintomas em profissionais que tocam instrumentos de sopro e corda, como pode ser observado na Tabela 3. 
Tabela 3. Distribuição do número de Músicos da OSUEL que apresentaram sintomas músculo-esqueléticos, nos sete dias e nos doze meses precedentes à auto-aplicação do questionário e tipo de instrumento.

\begin{tabular}{|c|c|c|c|c|c|c|c|c|c|}
\hline \multirow{4}{*}{ INSTRUMENTO } & \multicolumn{8}{|c|}{ SINTOMAS MÚSCULO-ESQUELÉTICOS } & \multirow{4}{*}{ Total } \\
\hline & \multicolumn{4}{|c|}{ ÚLTIMOS DOZE MESES } & \multicolumn{4}{|c|}{ ÚLTIMOS SETE DIAS } & \\
\hline & \multicolumn{2}{|c|}{\begin{tabular}{|l|} 
SIM \\
\end{tabular}} & \multicolumn{2}{|l|}{ NÃO } & \multicolumn{2}{|c|}{ SIM } & \multicolumn{2}{|l|}{ NÃ̃O } & \\
\hline & $\mathrm{n}$ & $\%$ & $\mathrm{n}$ & $\%$ & $\mathrm{n}$ & $\%$ & $\mathrm{n}$ & $\%$ & \\
\hline Corda & 18 & 81,8 & 4 & 18,2 & 17 & 71,3 & 5 & 22,7 & 22 \\
\hline Sopro & 9 & 100,0 & - & - & 7 & 77,8 & 2 & 22,2 & \\
\hline Metal & 5 & 50 & 5 & & 5 & 50,0 & 5 & 50,0 & 10 \\
\hline Outros & 3 & 75,0 & 1 & 25,0 & 3 & 75,0 & 1 & 25,0 & \\
\hline
\end{tabular}

Músicos estão expostos não somente a cargas físicas, mas também a cargas emocionais, em decorrência da própria atividade, como interpretar diante do público, sob olhar constante, cuidadoso e crítico dos regentes e cobrança da perfeição de si próprio (STERNBACH, 1993). Além disso, para atingir uma performance excelente, necessitam de uma rotina diária com treinamento individual, ensaios com os demais componentes e cuidados com a alimentação e descanso. $\mathrm{O}$ treinamento individual requer muitas horas de isolamento o que pode levar a uma perda de identidade com a sociedade. No treinamento em grupo, os músicos devem coordenar a sua performance com os demais integrantes. Observase uma obsessão pelo trabalho e toda essa rotina pode ser causadora de estresse (FETTER, 1993).

A atividade profissional do músico apresenta um padrão de trabalho não-usual e desse modo a vida familiar também fica comprometida, uma vez que as apresentações necessitam de viagens e com frequiência as apresentações ocorrem em finais de semana (FETTER, 1993).

Ribeiro (1997) e Sato (2001) referem existir uma forte associação entre sintomas músculo-esqueléticos e sofrimento psíquico. A maioria dos estudos mostra uma associação entre pelo menos um fator psicossocial relacionado ao trabalho e o aparecimento de sintomas em membros superiores. $\mathrm{O}$ estresse no trabalho foi consistentemente associado às desordens de membros superiores (BONGERS; KREMER; TER LAAK, 2002).

Diante de toda essa carga física e mental de trabalho dos músicos, fica fácil entender por que esses profissionais adoecem, porém é difícil identificar o maior responsável por tanto sofrimento.

Dos 32 músicos que referiram ter apresentado sintomas músculo-esqueléticos nos últimos sete dias, $14(43,8 \%)$ relataram ter realizado algum tratamento: tendo $8(25,0 \%)$ optado por tratamento médico, 9 $(28,1 \%)$ por tratamento de fisioterapia e $2(6,3 \%)$ por autotratamento. Os Músicos relataram ter procurado mais de um tratamento, por este motivo o número e o percentual apresentado correspondem às respostas obtidas e não ao número da amostra. Cinco músicos $(15,6 \%)$ utilizaram medicamentos para o tratamento dos sintomas, $5(15,6 \%)$ realizaram repouso ou utilizaram órteses, e $1(3,1 \%)$ houve necessidade de cirurgia.

De acordo com Dupuis (1993), muitos músicos só procuram assistência médica em casos já bem avançados e o tratamento nessas situações requer um afastamento prolongado do instrumento, fato não facilmente aceito. Segundo Nourissat, Chamagne e Dumontier (2003), do total de 277 músicos que procuraram o serviço médico, mais da metade apresentavam queixas de dor, $18 \%$ porque perceberam algum problema quando estavam tocando e somente $17 \%$ para esclarecimentos.

Em decorrência dos sintomas músculo-esqueléticos apresentados, 15 (33,3\%) músicos relataram ter perdido dias de trabalho, nos últimos doze meses, sendo que 7 $(15,6 \%)$ se afastaram no período de um a sete dias e $8(17,8 \%)$ acima de trinta dias.

Os músicos relataram ainda que deixaram de realizar outras atividades como: $4(8,8 \%)$ pentear o cabelo, cortar unhas, pegar objetos; $8(17,8 \%)$ serviços domésticos; $8(17,7 \%)$ lazer e atividade física e $18(40,0 \%)$ na atividade profissional. As principais mudanças foram em relação a troca de assento, realização de intervalos, cuidado com a postura e redução da carga horária de treinamento.

\section{Considerações Finais}

Os dados demonstraram que os músicos pesquisados apresentaram elevados índices de 
sintomas músculo-esqueléticos. Esses sintomas podem estar relacionados às cargas físicas e emocionais da própria atividade. Diante dos resultados encontrados, faz-se necessária a elaboração e implantação de estratégias para amenizar a carga de trabalho e evitar maiores agravos.

\section{Referências}

AMADIO, P. C.; RUSSOTTI, G. M. Evaluation and treatment of hand and wrist disorders in musicians. Hand Clinics, Philadelphia, v.6, n.3, p.405-416, 1990.

BEJJANI, F. J; KAYE G. M; BENHAM M. Musculoskeletal and neuromuscular conditions of instrumental musicians. Archives of Physical Medicine Rehabilitation,, Philadelphia, n.77, p.406-413, 1996.

BONGERS, P. M; KREMER, A. M; TER LAAK, J. Are psychosocial factors, risk factors for symptoms and signs of the shoulder, elbow, or hand/wrist?: a review of the epidemiological literature. American Journal of Industrial Medicine, New York, v.41, n.5, p.315-342, maio, 2002.

BRASIL. Secretaria de Políticas de Saúde. Departamento de Ações Programáticas e Estratégias. Protocolo de investigação, diagnóstico, tratamento e prevenção de lesões por esforços repetitivos distúrbios: osteomusculares relacionados ao trabalho. Brasília: Ministério da Saúde, 2000. 32p.

BRANDFONBRENER, A. G. The epidemiology and prevention of hand and wrist injuries in performing artists. Hand Clinics, Philadelphia, v.6, n.3, p.365-377, 1990.

BRITO, A C.; ORSO, M. B; GOMES, E. Lesões por esforços repetitivos e outros acometimentos reumáticos em músicos profissionais. Revista Brasileira de Reumatologia, São Paulo, v.32, n.2, p. 79-83, 1992.

DUPUIS, M. Pathologies of the musculo-skeletal system in musicians. Union Medicale du Canada, Montreal, v.122, n.6, p.432-436, 1993.

FETTER, D. Life in the orchestra. Maryland Medical Journal, Baltimore, v.42, n.30, p.289-292, 1993.

FISHBEIN, M.; MIDDLESTADT, S. E; OTTATI, V.; STRAUS, S.; ELLIS, A. Medical problems among ICSOM musicians: overview of a national survey. Medical Problems of Performing Artists, Philadelphia, v.3, p.1-8, 1988.

FRY, H. J. H. Overuse syndrome of the upper limb in musicians. Medical Jounal of Australia, North Sydney, v.144, n.4, p.182-183, 1986.
HOPPMANN, R. A. Instrumental musicians' hazards. Occupational Medicine, Philadelphia, v.16, n.4, p.619-631, oct./dec., 2001.

JOUBREL, I.; ROBINEAU, S.; PÉTRILLI, S.; GALLIEN, P. Musculoskeletal disorders in instrumental musicians: epidemiological study. Annales de Réadaptation et de Médecine Physique, Paris, v.44, n.2, p.72-80, 2001.

KRAPAC, L. The most common overuse injury syndromes of the upper extremity associated with work activity. Arhiv za Higijenu Rada i Toksikologiju, Zagreb, v.52, n.4, p.41520,2001.

KUORINKA, I.; JONSSON, B.; KILBOM, A. Standardised Nordic questionnarie for the analysis of Musculoskeletal symptoms. Applied Ergonomics, Kidlington,v.18, p.233237, 1987.

LIU, S.; HAYDEN, G. Maladies in musicians. Southern Medical Journal, Birmingham, v.95, n.7, p.727-734, 2002.

LOCKWOOD, A. H. Medical problems of musicians. The New England Journal of Medicine, Boston, v.320, n.4, p.221-227, 1989.

NOURISSAT, G.; CHAMAGNE, P.; DUMONTIER, C. Reasons why musicians consult hand surgeons. Revue de Chirurgie Orthopedique et Reparatrice de L'Appareil Moteur, Paris, v.89, n.6, p.524-531, oct., 2003.

PARASURAMAN, S.; PUROHIT, Y. S. Distress and boredom among orchestra musicians: the two faces of stress. Journal of Occupational Health Psychology, Baltimore, v.5, n.1, p.74-83, 2000.

PINHEIRO, F. A.; TRÓCCOLI, B. T; CARVALHO, C. V. Validação do Questionário Nórdico de sintomas osteomusculares como medida de morbidade. Revista de Saúde Pública, São Paulo, v.36, n.3, p.207-212, 2002.

RANNEY, D. Distúrbios osteomusculares crônicos relacionados ao trabalho. São Paulo: Rocca, 2000. 344p.

RIBEIRO, H. P. Lesões por Esforços Repetitivos (LER): uma doença emblemática. Cadernos de Saúde Pública, Rio de Janeiro,v.13, supl. 2, p.85-93, 1997.

ROSETY-RODRIGUES, M. The influence of the active range of movement of pianist's wrists on repetitive strain injury. European. Journal of Anatomy, Salamanca, v.7, n.2, p.75-77, 2003.

SATO, L. LER: objeto e pretexto para a construção do campo trabalho e saúde. Cadernos de Saúde Pública, Rio de Janeiro, v.17, n.1, p.147-152, 2001.

SIEMON, B.; BORISCH, N. Problems of the musculoskeletal system in amateur orchestra musicians under special consideration of the hand and wrist. Handchirurgie Mikrochirurgie Plastishe Chirurgie, Stuttgart, v.34, n.2, p.89-94, mar., 2002. 
SOARES, J. F.; SIQUEIRA, A. L. Introdução à estatística médica. Belo Horizonte: Departamento de Estatística, UFMG, 1999.300p.

STERNBACH, A. C. S. W. Adressing stress-related ilness in professinal musicians. Maryland Medical Journal, Baltimore, v.42, n.3, p.283-288, 1993.

TOLEDO, S. D.; AKUTHOTA, V.; DRAKE, D. F.; NADLER, S.F. et al. Sports and performing arts medicine. 5. Issues relating to musicians. Archives of Physical Medicine Rehabilitation, Philadelphia, v.85, n.3, suppl. 1, p.72-74, mar., 2004.
ZAZA, C. Playing-related musculoskeletal disorders in musicians: a systematic review of incidence and prevalence. Canadian Medical Association Journal, Ottawa, v.158, n.8, p.1019-1025, 1998.

ZAZA, C.; CHARLES, C.; MUSZYNSKI, A. The meaning of playing-related musculoskeletal disorders to classical musicians. Social Science and Medicine, Oxford, v.47, n.12, p. 2013-2023, 1998. 
